\title{
Hydroxyl radical production and storage in analogues of amorphous interstellar silicates: a possible "wet" accretion phase for inner telluric planets
}

\author{
Z. Djouadi ${ }^{1}$, F. Robert ${ }^{2}$, L. Le Sergeant d'Hendecourt ${ }^{1}$, S. Mostefaoui ${ }^{2}$, H. Leroux ${ }^{3}$, A. P. Jones ${ }^{1}$, and J. Borg ${ }^{1}$ \\ ${ }^{1}$ Institut d'Astrophysique Spatiale (IAS), Bâtiment 121, Université Paris-Sud 11 and CNRS, 91405 Orsay, France \\ e-mail: zahia.djouadi@ias.u-psud.fr \\ 2 Muséum National de l'Histoire Naturelle, UMR 7202, 75005 Paris, France \\ 3 Unité Matériaux et Transformations, UMR 8207, Université Lille 1 and CNRS, 59655 Villeneuve d'Ascq, France
}

Received 15 February 2011 / Accepted 29 April 2011

\begin{abstract}
Context. Interstellar silicate grains are thought to be amorphized by interaction with high- and low-energy particle interactions in astrophysical environments. In addition, low energy (a few $\mathrm{keV}$ ) particles will implant atoms within the grains.

Aims. In this paper we experimentally investigate the consequence of the implantation of $\mathrm{H}^{+}$at low irradiation energies into analogues of interstellar silicate grains, and look for the formation of hydroxyl radicals within the silicate matrix.

Methods. Thin amorphous silicate films $(\sim 100 \mathrm{~nm})$ were sequentially irradiated with $\mathrm{H}^{+}$ions at low energies $(3.5,2.5$ and then $1.5 \mathrm{keV}$ ) ensuring an implantation of the ions through the full depth of the films. The fluences used, $3 \times 10^{16}, 10^{17}$ and $3 \times 10^{17} \mathrm{H}^{+} / \mathrm{cm}^{2}$, are compatible with those expected in shocks in the interstellar medium. We used infrared spectroscopy to monitor and quantify the $\mathrm{OH}$ band evolution after irradiation. In order to distinguish the newly formed $\mathrm{OH}$ groups from those originating from unavoidable atmospheric contamination, the $\mathrm{D} / \mathrm{H}$ depth ratios were measured with a NanoSIMS ion microprobe.

Results. An increase in the $\mathrm{OH}$ band strength in the infrared spectra after irradiation reveals the formation of $\mathrm{OH}$ bonds within the irradiated silicate thin films. NanoSIMS measurements of the $\mathrm{D} / \mathrm{H}$ signature in the region of ion implantation show that the newlyformed $\mathrm{OH}$ groups make up about $40 \%$ of the observed $\mathrm{OH}$ band in the IR, the rest are due to an atmospheric hydroxylation of the sample. Only about $2 \%$ of the incident ions lead to $\mathrm{OH}$ bond formation and, at most, the irradiated silicates retain about $3 \%$ of the incident protons as $\mathrm{OH}$ groups within their structure.

Conclusions. Our laboratory experimental simulations show a possible production and storage of hydroxyl radicals in amorphous laboratory silicates. In the astrophysical context, such $\mathrm{OH}$ radicals, strongly bonded to pre-accretion material, could constitute a non negligible reservoir of - $\mathrm{OH}$, thus water. These experimental results allow us to revisit and reinstate the hypothesis of a possible "wet" accretion of the telluric planets early in the history of the formation of the Solar System.
\end{abstract}

Key words. astrochemistry - methods: laboratory

\section{Introduction}

Spectroscopic results from the InfraRed Astronomical Satellite (IRAS), InfraRed Space Observatory (ISO) and more recently, Spitzer Space Telescope, reveal that the nature of the silicate dust varies as a function of the astronomical environment. Whereas crystalline silicates are present at the beginning and the end of the dust cycle, very little $(<2 \%)$ crystalline silicate dust, if any, is found in the diffuse interstellar medium (ISM), which correspond to that phase in the dust life cycle where grains spend most of their time (e.g., Li \& Draine 2001; Kemper et al. 2004). The presence of crystalline silicates in the dust shells of evolved stars ("young dust, old stars") and early-type stars ("old dust, young stars") was reported by Waters et al. (1996), Waelkens et al. (1996), Sylvester et al. (1999) and Molster et al. (2002). The first astronomical evidence for the presence of crystalline silicates in comets was reported by Bregman et al. (1987) and confirmed by Campins et al. (1989) with the detection of fine structure on the $10 \mu \mathrm{m}$ IR silicate feature of the comet P/Halley dust. Later, abundant crystalline olivine rich silicates in the comet Hale-Bopp were seen by ISO (Crovisier et al. 1997). Other comet encounter missions; Giotto (Halley comet in 1986 and
26P/Grigg-Skjellerup in 1992) and Deep Impact (Tempel 1 in 2005) revealed the nature of the silicates present in the encountered (or impacted for Tempel 1) comets. Finally, the Stardust mission collected thousands of particles from comet 81P/Wild2 and brought them back to Earth in 2006, this allowed for the first time the study and analyses of cometary grains of a known origin in the laboratory (Brownlee et al. 2006).

Since dust is formed around evolved stars and injected into the interstellar medium by stellar winds, the astronomical observations indicate that silicates become fully amorphized during their transit through the ISM. Among the processes that could account for the disappearance of the crystalline silicates in the diffuse ISM, dust amorphization induced by ion irradiation in supernovae shock waves in the warm ISM appears to be the most efficient process as demonstrated by dedicated laboratory experiments (Demyk et al. 2001; Carrez et al. 2002; Demyk et al. 2004). During the propagation of shock waves, the interstellar gas is ionized and the gas and dust acquire relative velocities leading to dust irradiation by gas, principally protons (e.g. Jones et al. 1994, 1996). Furthermore, the size distribution of the dust is modified by destruction and shattering leading to a tranfer of large grains to small grains (e.g. Jones et al. 1996). 
In addition, as noted by Demyk et al. (2001) sputtering by low energy $\mathrm{He}^{+}$ions progressively reduces the $\mathrm{O} / \mathrm{Si}$ and $\mathrm{Mg} / \mathrm{Si}$ ratios of the samples, thus leading to silicates of a more pyroxenetype composition a trend which is indeed compatible with what is observed in the ISM (Demyk et al. 2001). In high velocity shocks $\left(v \geq 100 \mathrm{~km} \mathrm{~s}^{-1}\right)$, the ionized gas reaches energies on the order of one to tens of $\mathrm{keV}$. The column of gas accelerated in such shocks, which corresponds to the irradiation fluence, is of the order of $10^{16}-10^{18} \mathrm{H}^{+} / \mathrm{cm}^{2}$, for 5 to $250 \mathrm{~nm}$ radius-grains (Jones et al. 1996). In this irradiation regime nuclear interactions with the target atoms are dominant and the grains can be amorphized as shown in various simulation experiments (Demyk et al. 2001; Carrez et al. 2002; Jäger et al. 2003a; Brucato et al. 2004; Djouadi et al. 2005). In addition, the grain sizes are of the same order as the implantation range of the ions, leading to the complete amorphization of the grains. In the laboratory, ion implantation (e.g. $\mathrm{He}^{+}$and $\mathrm{Ar}^{+}$) is responsible for the formation of bubbles within the irradiated material (Carrez et al. 2002; Jäger et al. 2003a; Demyk et al. 2004) and may contribute to the build-up of grain porosity that could play a role in subsequent gas-grain surface interactions. Note that this porosity is also compatible with the observed optical behavior of the grains where some porosity has to be added in grain model calculations to better fit the silicate band at 9.7 microns (Mathis 1998).

Implantation may also lead to the formation of new chemical bonds within the grains if the implanted ions are themselves reactive $\left(\mathrm{e} . \mathrm{g} . \mathrm{H}^{+}\right)$. The effect of the implantation of reactive species has not yet been studied in depth in the astrophysical context of the evolution of interstellar grains. For example, a shock wave which accelerates the gas to energies of a few $\mathrm{keV}$, with a fluence of $10^{18}$ ions $/ \mathrm{cm}^{2}$, would implant almost all of the incident protons within a $100 \mathrm{~nm}$ grain. In the case of full implantation, the $\mathrm{H}: \mathrm{O}$ ratio (in the silicates) would be close to 1 . However, a large part of the hydrogen probably desorbs from the grains as $\mathrm{H}_{2}$ molecules but some may remain as interstitials in the silicate network and then react to form $\mathrm{OH}$ and/or $\mathrm{SiH}$ chemical bonds.

Observations of silicates containing $\mathrm{OH}$ species in astrophysical environments are scarce because their spectroscopic signatures coincide with those of well known dust compounds such as amorphous olivine and pyroxene for the $\mathrm{SiO}$ stretching and bending modes at $\sim 9.8$ and $\sim 18 \mu \mathrm{m}$ and water ice for the $\mathrm{OH}$ stretching modes which arises in the $2.7-3.5 \mu \mathrm{m}$ wavelength range. Tentative detections have been reported, but not confirmed for the diffuse ISM by Whittet et al. (1997) via the $\mathrm{OH}$ stretching mode at $\sim 2.75 \mu \mathrm{m}$. Timmermann \& Larson (1993) observed a sharp absorption edge at $2.7 \mu \mathrm{m}$ in the near IR spectrum of SN 1987A. They attribute this feature to structural OH in silicate minerals in circumstellar dust ejected from the progenitor star during its red supergiant phase, but no corresponding $9.7 \mu \mathrm{m}$ silicate feature in SN 1987A has been observed at any time. $\mathrm{OH}$ bearing silicates have also been proposed to explain a band at $\sim 100 \mu \mathrm{m}$ observed in emission in the spectrum of the young star HD 142527 (Malfait et al. 1999). Interestingly, hydroxylated amorphous silicates are found within interplanetary dust particles (IDPs) in the GEMS (glass with embedded metal and sulphides; Thomas et al. 1993; Bradley 1994; Bradley et al. 2005). Some of these GEMS are believed to be presolar (Messenger et al. 2003) and to originate from the ISM (Bradley et al. 1999). The presence of hydroxyl groups in these amorphous silicates has been tentatively interpreted as a result of hydrogen implantation by irradiation processes (Bradley 1994).

In this study we examine the consequences of the irradiation of amorphous silicates (olivine-type) with low energy $\mathrm{H}^{+}$ions within the implantation energy regime. The aim of this study was to determine whether the implanted protons can form chemical bonds with the target atoms, to estimate the abundances of the formed and stored $\mathrm{OH}$ in the silicate network, and to evaluate this as a potential source of water, within the framework of the debated ideas on the possibility of "wet" accretion of the inner telluric planets such as the Earth (Drake 2005) as opposed to "dry" accretion models (Boss 1998).

\section{Experimental methods}

We used thin amorphous silicate films $(\sim 100 \mathrm{~nm}$ thick $)$ as analogs of interstellar silicate dust. The samples were irradiated with protons at energies and fluences compatible with those expected in interstellar shock waves, to ensure ion implantation commensurate with the sample thicknesses. The irradiated samples were first characterized by IR spectroscopy to study the structural and/or chemical modifications and search for hydrogen-silicate bonds in particular $\mathrm{OH}$ and $\mathrm{SiH}$ bonds.

Because sample porosity is induced by irradiation, possible contamination reactions with atmospheric moisture leading to $\mathrm{OH}$ bonds are likely and difficult to avoid. In order to estimate the relative fraction of $\mathrm{OH}$ originating from atmospheric water and from protons recombined with $\mathrm{SiO}$ during the irradiation sequence, we performed NanoSIMS measurements in order to discriminate between the two populations. The implanted protons are isotopically labeled compared with those from atmospheric water, since they are deuterium-free $(\mathrm{D} / \mathrm{H}=0)$ because they were selected by a mass spectrometer, whereas terrestrial water contains $0.014 \%$ of $\mathrm{D}$ relative to $\mathrm{H}\left(\mathrm{D} / \mathrm{H}=(140 \pm 10) \times 10^{-6}\right.$. Such a difference in isotopic composition is accessible with the NanoSIMS ion probe. Depth profiling of hydrogen concentration and isotopic composition within the samples were performed with the NanoSIMS.

Furthermore in order to check the chemical stability of the $\mathrm{OH}$ groups newly-formed by a reaction between the amorphous silicate matrix and the implanted $\mathrm{H}$ atoms, Almax 3 was heated to $750{ }^{\circ} \mathrm{C}$ under vacuum before the NanoSIMS measurements. Such a treatment should lead to the outgasing of implanted $\mathrm{H}$ atoms while enhancing the crystallization of the matrix and the stabilization of the hydroxyl groups.

\subsection{Sample preparation}

The thin films, typically $100 \mathrm{~nm}$ thick, were prepared by electron beam evaporation and cold condensation onto $3 \mathrm{~mm}$ diameter diamond discs (see Djouadi et al. 2005, for details). This geometry ensures a high surface-to-volume ratio compatible with interstellar dust. The San Carlos $\mathrm{Mg}$ rich composition olivine $\left(\mathrm{Mg}_{1.8} \mathrm{Fe}_{0.19} \mathrm{Ni}_{0.01} \mathrm{SiO}_{4}\right)$ was used as a precursor.

To distinguish the four samples used in this study we will, in the following, label our samples Almax\#. (Almax is the company that provided the diamond discs.)

\subsection{Sample irradiation}

The sample irradiation was performed at the isotope separator facility, SIDONIE, in Orsay (Chauvin et al. 2004). The proton beam current was kept below $10 \mu \mathrm{A}$ in order to avoid excessive heating of the samples. The irradiation was performed under vacuum $\left(10^{-7} \mathrm{mbar}\right)$ and at room temperature. Different proton fluences were used to study the effect of the dose on the $\mathrm{OH}$ formation in the silicates. The fluences used were $3 \times 10^{16}, 10^{17}$ and $3 \times 10^{17} \mathrm{at} / \mathrm{cm}^{2}$. Each sample was sequentially irradiated 
Table 1. The studied samples and their irradiation histories.

\begin{tabular}{lccc}
\hline \hline $\begin{array}{l}\text { Sample } \\
\text { reference }\end{array}$ & $\begin{array}{c}\text { Proton energies } \\
(\mathrm{keV})\end{array}$ & $\begin{array}{c}\text { Fluence per energy } \\
\left(\mathrm{H}^{+} / \mathrm{cm}^{2}\right)\end{array}$ & $\begin{array}{c}\text { Total fluence } \\
\left(\mathrm{H}^{+} / \mathrm{cm}^{2}\right)\end{array}$ \\
\hline Almax1 & $3.5,2.5$ and 1.5 & $10^{16}$ & $3 \times 10^{16}$ \\
Almax2 & $3.5,2.5$ and 1.5 & $3.3 \times 10^{16}$ & $\sim 10^{17}$ \\
Almax3 & $3.5,2.5$ and 1.5 & $10^{17}$ & $3 \times 10^{17}$ \\
Almax4 & No irradiation & None & None \\
\hline
\end{tabular}

with three different incident energies 3.5, 2.5 and then $1.5 \mathrm{keV}$ in order to implant the ions over a range of depths in the samples. The ion distributions in the samples were estimated using the Monte-Carlo TRIM simulation code (www.srim.org). The average penetration depths of $\mathrm{H}^{+}$at energies of 1.5, 2.5 and $3.5 \mathrm{keV}$ are respectively 32, 48 and $64 \mathrm{~nm}$ (assuming a density of $2.3 \mathrm{~g} / \mathrm{cm}^{3}$ for the amorphous olivine). Table 1 summarizes the experimental irradiation conditions for each sample.

We also used a "blank" as a "control sample", noted as Almax4 (see Table 1) in order to evaluate the unavoidable contribution of the atmospheric water on the $\mathrm{OH}$ band and its evolution during and/or between the different steps in the experiments. To prevent and minimize atmospheric water contamination we systematically preserved the samples under primary vacuum $\left(\sim 10^{-2} \mathrm{mbar}\right)$ in a desiccator.

\subsection{Sample analyses}

\subsubsection{Infrared spectroscopy}

Infrared spectroscopy (IR) is a sensitive tool for the study of the structural and chemical modifications in the analyzed samples and it also allows a direct comparison with astronomical observations. IR measurements were performed with a Bruker VERTEX 70 IR Fourier transform interferometer. The configuration used consisted of a Globar (black body-like) source coupled with a $\mathrm{KBr}$ beamsplitter and a deuterated triglycine sulfate (DTGS) room temperature detector. The final transmittance spectra were obtained in the range $4000-400 \mathrm{~cm}^{-1}(1.67-25 \mu \mathrm{m})$ by averaging five spectra of 512 scans at a spectral resolution of $4 \mathrm{~cm}^{-1}$.

\subsubsection{NanoSIMS analyses}

NanoSIMS (secondary ion mass spectrometry) provides quantitative information on the elemental and isotopic spatial distributions, in our case, $\mathrm{H}$ and $\mathrm{D}$, within the samples. The spatial lateral resolution of the instrument is of the order of $100 \mathrm{~nm}$. In addition, since the technique is based on the slow pulverization of the sample surface, it is also possible to perform depth profiling for which the chemical and the isotopic compositions can be recorded with a depth resolution of a few atomic layers.

In the present experiment, the measurement of the hydrogen isotopic composition $(\mathrm{D} / \mathrm{H}$ ratio) within the irradiated samples allows us to determine to what extent the $\mathrm{OH}$ groups detected by infrared spectroscopy correspond to implanted H. Indeed, the contamination of the irradiated samples with atmospheric moisture remains a priori a possibility. For the irradiation experiments, the incoming beam of ions was deflected by a mass spectrometer, the $\mathrm{D} / \mathrm{H}$ ratio of implanted hydrogen is zero. On the contrary, the terrestrial moisture has a $\mathrm{D} / \mathrm{H}$ ratio lying in the domain $(130-150) \times 10^{-6}$. It is therefore possible to discriminate implanted $\mathrm{H}$ from $\mathrm{OH}$ atmospheric contamination and to calculate the relative fraction of the two contributions.
NanoSIMS analyses, performed at the Muséum National de l'Histoire Naturelle (MNHN) in Paris, were conducted on the samples Almax2, Almax3 and Almax4, using the National Facility Cameca NanoSIMS N50. The samples were coated with gold to ensure their charge conduction. The size of the analyzed area (i.e. the area rastered by the primary $\mathrm{Cs}^{+}$ions) was $15 \times 15 \mu \mathrm{m}^{2}$ and several areas were analyzed in the same sample. In series of analytical sessions we measured (i) $\mathrm{D}$ and $\mathrm{H}$ and (ii) ${ }^{29} \mathrm{Si},{ }^{13} \mathrm{C}$ and $\mathrm{H}$. The measurements of $\mathrm{Si}$ and $\mathrm{C}$ allowed us to locate the silicate/diamond interface, diamond being the substrate. Secondary ions were measured in multi-collection mode on electron multipliers. The primary current was $250 \mathrm{pA}$ (picoAmps) yielding the complete removal by sputtering of a $\sim 100 \mathrm{~nm}$ thick sample in $\sim 200 \mathrm{~s}$. At such high spatial depth resolution the removal of the sample by the primary ion beam is not spatially homogeneous. In other words, in real time, the carbon surface underneath the sample layer appears in the ion images as individual spots during the progressive removal of the sample layer.

\subsection{Data analyses}

\subsubsection{IR data analyses}

For each sample we obtained three principal raw spectra. The first spectrum gives the diamond substrate IR signatures, the second is obtained after the olivine film deposition and the third is obtained after the $\mathrm{H}^{+}$irradiation step. To obtain the olivine film spectrum before and after irradiation we divided the second and third spectra by the first spectrum.

Our IR study which is essentially based on the analyses and comparison of the spectra before and after irradiation consists of the evaluation of the column density of the $\mathrm{OH}$ band. For this purpose, after dividing each raw spectrum, obtained before and after irradiation, by its corresponding substrate spectrum, we extract the IR data in the range $3800-3000 \mathrm{~cm}^{-1}$ for the $\mathrm{OH}$ band and subtract a linear baseline over this range. The same procedure is applied to the $\mathrm{SiO}$ band studied in the range 1300 $700 \mathrm{~cm}^{-1}$.

The column density $N$, in $\mathrm{cm}^{-2}$, is then estimated using the classical formula (d'Hendecourt \& Allamandola 1986):

$N=\int \frac{\tau \mathrm{d} v}{A}$

where $\tau$ is the optical depth related to the transmittance $\mathrm{T}$ by the relationship $\tau=-\ln T, v$ is the frequency in $\mathrm{cm}^{-1}$ and $A$ is the band strength in $\mathrm{cm} \mathrm{mol}^{-1}$. The values used in this work are $2.8 \times 10^{-16} \mathrm{~cm} \mathrm{~mol}^{-1}$ and $2.0 \times 10^{-16} \mathrm{~cm} \mathrm{~mol}^{-1}$ for the stretching modes of the $\mathrm{OH}$ and $\mathrm{SiO}$ bonds respectively (Matrajt et al. 2005, and references therein).

We also searched for the $\mathrm{SiH}$ stretching band with a peak position in the 2278 to $2110 \mathrm{~cm}^{-1}$ region (Moore et al. 1991; Nuth et al. 1992). For this, we extracted the data in the range from 1800 to $2300 \mathrm{~cm}^{-1}$. The results are discussed below in the Results section.

\subsubsection{NanoSIMS data analyses}

For each rastered area up to 50 ion images $\left(15 \times 15 \mu \mathrm{m}^{2}\right)$ were recorded and the total number of $\mathrm{H}^{+}$and $\mathrm{D}^{+}$ions constituting these images were used to calculate what is here referred to as the instrumental $\mathrm{D} / \mathrm{H}$ ratio: $(\mathrm{D} / \mathrm{H})_{\text {Instr. }}$ Backgrounds on the masses $1(\mathrm{H})$ and 2 (D) were negligibly small. However during the pulverization process and during the transfer of the ions 
emitted from the surface of the sample to the mass spectrometer, a series of isotopic fractionation effects may take place. The overall effect $\alpha_{\text {Instr }}$ is expressed as:

$\alpha_{\text {Instr }}=\frac{\left(\frac{\mathrm{D}}{\mathrm{H}}\right)_{\text {Instr }}}{\left(\frac{\mathrm{D}}{\mathrm{H}}\right)_{\text {Real }}}$

The subscript "Instr" designates the fact that this effect is entirely caused by the analytical protocol of the extraction and ion detections. Note that the parameter $\alpha_{\text {Instr }}$ encompasses variations due to the nature of the material constituting the sample; this effect is referred to as the "matrix effect". In this work the instrumental fractionation factor $\left(\alpha_{\text {Instr }}\right)$ is measured from the results obtained on the two depth profiles from Almax4, i.e. from the non irradiated sample. Although the absolute $\mathrm{D} / \mathrm{H}$ ratio of this sample, $(\mathrm{D} / \mathrm{H})_{\text {Real }}$, is not accurately known, it must lie within the usual terrestrial values for bound water; that is between $130 \times 10^{-6}$ and $150 \times 10^{-6}$. This gives $1.0 \leq \alpha_{\text {Instr }} \leq 1.1$. Although, the corresponding error bars on such a range for $\alpha_{\text {Instr }}$ are quite large (for usual geochemical analyses), they are commensurate with the isotope variations that we observed for the irradiated samples Almax 2 and Almax3. Therefore we did not attempt to measure more precisely the matrix effect linked to the amorphous nature of olivine.

Each ion image is recorded after $5 \mathrm{~s}$ of sputtering, called a cycle. The first $\sim 10$ cycles (surface water) are eliminated from the calculation of the bulk $\mathrm{D} / \mathrm{H}$ ratio because of the low count rates for $\mathrm{H}$ which induce large uncertainties in the $\mathrm{D} / \mathrm{H}$ ratio. After cycle \#40, the hydrogen signal decreased by a factor of $\sim 50$ relative to its maximum, indicating that most of the sample has been sputtered away.

The depth profiling for $\mathrm{H}$ within the samples Almax 2 and Almax 3 are reported in Figs. 6 and 7, respectively, along with their corresponding $\mathrm{D} / \mathrm{H}$ ratio. The precision on the $\mathrm{D} / \mathrm{H}$ ratio reported as error bars ( 2 sigma) in Figs. 6 and 7 is the statistical error i.e. $2 \sigma= \pm 2 / \sqrt{n}$ with $n$ the total number of deuterium ions detected by the collector. The $\mathrm{D} / \mathrm{H}$ ratio integrated over the variations of $\mathrm{H}$, are reported in Table 3 for the two analyzed samples.

From these data, the fraction of $\mathrm{H}$ originating from atmospheric moisture can be calculated from the mass balance:

$\left(\frac{\mathrm{D}}{\mathrm{H}}\right)_{\text {Sample }}=\boldsymbol{x}\left(\frac{\mathrm{D}}{\mathrm{H}}\right)_{\text {Moisture }}+(1-x)\left(\frac{\mathrm{D}}{\mathrm{H}}\right)_{\text {Implanted-H }}$

$\boldsymbol{x}$, is the relative fraction of the atmospheric moisture $\mathrm{H}$ in the sample. Taking a minimum reasonable $\mathrm{D} / \mathrm{H}$ ratio for the atmospheric moisture of $125 \times 10^{-6}$, the value of $\boldsymbol{x}$ can be calculated for each data point since $(\mathrm{D} / \mathrm{H})_{\text {Implanted-H }}=0$. The values of $\boldsymbol{x}$ integrated over the whole depth profile are reported in Table 3.

In order to compare these $\boldsymbol{x}$ values with the contamination which is inherent to the sample, and present before irradiation, we calculated the hydrogen concentration ratio $\boldsymbol{R}=[\mathrm{H}]_{\text {Almax } 4} /[\mathrm{H}]_{\text {Sample }}$ where Almax 4 is the non-irradiated sample whose $\mathrm{H}$ concentration, $[\mathrm{H}]$, was also measured with the NanoSIMS (cf. Fig. 4). This ratio gives the relative proportion of $\mathrm{H}$ added after irradiation from both the incident protons and/or terrestrial moisture. Interestingly $\boldsymbol{R}$ and $\boldsymbol{x}$ are quite different, giving access to an estimate of the atmospheric contamination triggered by irradiation but not by the simple exposure to atmosphere.

Finally, we calculated the fluence of incident $\mathrm{H}^{+}$ions (expressed as $\mathrm{H}$ atoms $/ \mathrm{cm}^{2}$ in Table 3) implanted into the sample. This calculation has rather large uncertainties, principally due to

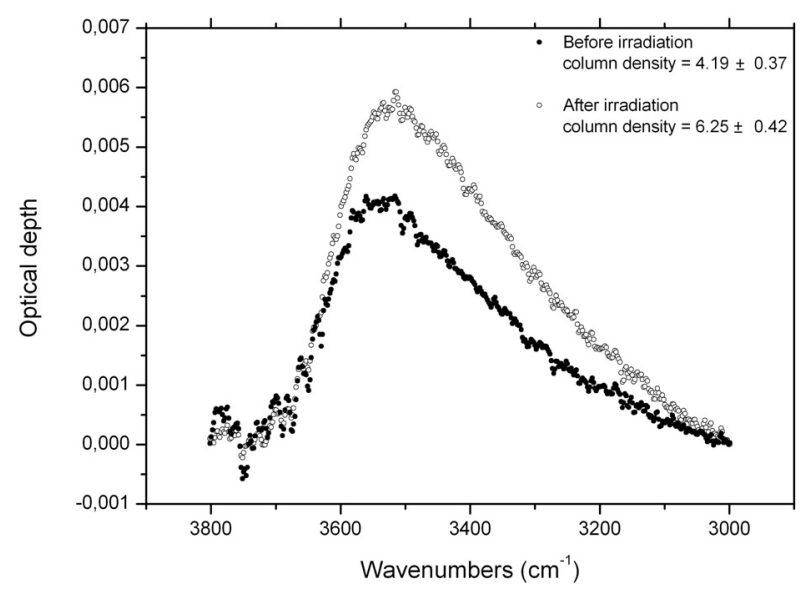

Fig. 1. The $\mathrm{OH}$ band plotted as optical depth versus wavenumber $\left(\mathrm{cm}^{-1}\right)$ for the sample Almax2, before (filled circles) and after (open circles) irradiation. In the upper right we give the $\mathrm{OH}$ column density (in units of $10^{15} \mathrm{OH} / \mathrm{cm}^{2}$ ). The spectra given here were obtained after a linear baseline correction.

the SIMS technology: i.e. the relative fraction of neutral atoms produced by sputtering but impossible to detect by mass spectrometry. The calibration of this effect was not possible because the small thickness of the amorphous silicate films prevents a determination of the absolute hydrogen concentration by simple chemical procedures, and hence a calculation of the ion to neutral ratio produced during sputtering. This neutral-ion conversion factor is generally 100/1 but conservatively, can lie between 50/1 and 300/1. In Table 3 we use an average value of $175 \pm 125$, this fluence is referred to as the "NanoSIMS C.D.".

\section{Results}

The main objective of these irradiation experiments on amorphous material was to estimate the consequences of proton implantation, the reactivity of $\mathrm{H}$ and its ability to form stable $\mathrm{OH}$ and/or $\mathrm{SiH}$ groups.

\subsection{OH IR band results}

Following the procedure described in Sect. 2.4.1, for each sample we plot the spectra obtained in the $\mathrm{OH}$ region. In Fig. 1, as an example, we compare the $\mathrm{OH}$ band of the Almax 2 sample before and after irradiation plotted as optical depth versus wavenumber. The $\mathrm{OH}$ band column density is given in the figure. An estimate of the error on the determination of the column density is obtained by varying the integration limits of the band by $\sim 50 \mathrm{~cm}^{-1}$ on each side.

Table 2 summarizes the $\mathrm{OH}$ column densities obtained for each sample before and after irradiation. The SiO column density is also tabulated for each sample after irradiation. The difference between the $\mathrm{OH}$ column densities in the sample before and after irradiation is also given.

Before irradiation all of the samples had an $\mathrm{OH}$ column density of $\sim 4.2 \times 10^{15} \mathrm{~cm}^{-2}$. As suggested by the blank sample Almax 4, this is likely due to surface atmospheric contamination, which was impossible to avoid despite all of the precautions taken during the experiments.

The $\mathrm{OH}$ column density of Almax4, the non irradiated sample, allows an estimation of the atmospheric contribution. It increased by about $10 \%$ throughout the experiment. However, this $\mathrm{OH}$ column density variation is clearly lower than those of the 


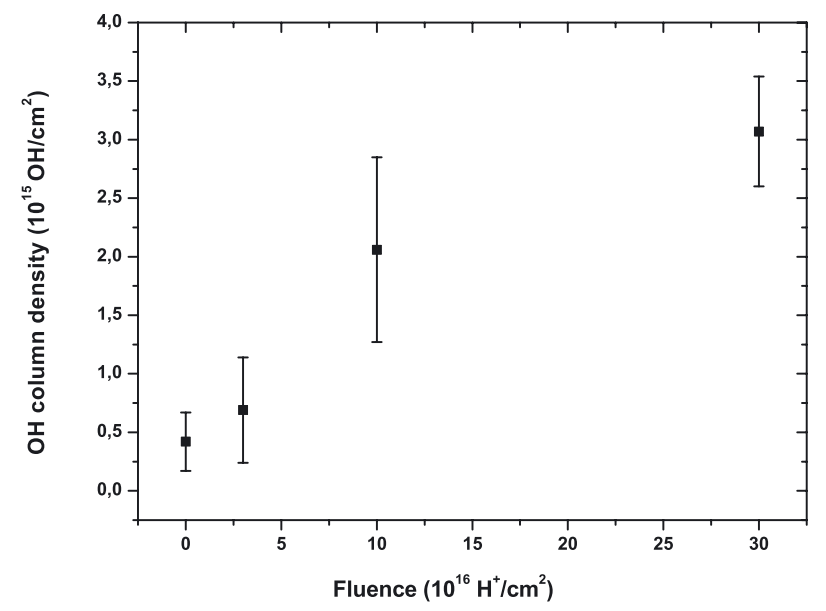

Fig. 2. The $\mathrm{OH}$ column densities (in units of $10^{15} \mathrm{OH} / \mathrm{cm}^{2}$ ) measured in the samples after irradiation; versus the fluence (in units of $10^{16} \mathrm{H}^{+} / \mathrm{cm}^{2}$ ). The point corresponding to a fluence of "0" gives the measured column density in the non-irradiated sample, Almax4.

Table 2. The $\mathrm{OH}$ column densities before and after irradiation, the difference $(\delta N(\mathrm{OH}))$, and the $\mathrm{SiO}$ column densities derived from the IR data.

\begin{tabular}{lcccc}
\hline \hline & \multicolumn{4}{c}{ Column densities (in units of $\left.10^{15} \mathrm{~cm}^{-2}\right)$} \\
Sample & $N(\mathrm{OH})_{\text {before }}$ & $N(\mathrm{OH})_{\text {after }}$ & $\delta N(\mathrm{OH})$ & $\mathrm{N}(\mathrm{SiO})$ \\
\hline Almax1 & $4.21 \pm 0.28$ & $4.90 \pm 0.17$ & $0.69 \pm 0.45$ & $104 \pm 4$ \\
Almax2 & $4.19 \pm 0.37$ & $6.25 \pm 0.42$ & $2.06 \pm 0.79$ & $108 \pm 4$ \\
Almax3 & $4.21 \pm 0.07$ & $7.28 \pm 0.40$ & $3.07 \pm 0.47$ & $94 \pm 4$ \\
Almax4 & $4.33 \pm 0.13$ & $4.75 \pm 0.12$ & $0.42 \pm 0.25$ & $100 \pm 4$ \\
\hline
\end{tabular}

irradiated samples, in particular compared with Almax2 ( 49\%) and Almax3 $(\sim 73 \%)$, and also Almax $1(\sim 16 \%)$ even considering the relatively large error bars associated with these measurements.

The changes in the $\mathrm{OH}$ column density in the irradiated samples clearly increases with increasing fluence (Fig. 2).

Considering the slope between the first two fluences $3 \times 10^{16}$ and $10 \times 10^{16} \mathrm{H}^{+} / \mathrm{cm}^{2}$, we obtain $0.196\left(\mathrm{OH} / \mathrm{H}^{+}\right)$. The slope between the two last fluences $10 \times 10^{16}$ and $30 \times 10^{16} \mathrm{H}^{+} / \mathrm{cm}^{2}$ is $0.051\left(\mathrm{OH} / \mathrm{H}^{+}\right)$. These values indicate that the number of $\mathrm{OH}$ obtained per $\mathrm{H}^{+}$decreases by a factor of $\sim 4$ when the fluence increases by a factor of 3 . This would support the idea of a saturation level of $\mathrm{OH}$ with increasing irradiation fluence.

Finally in the last column of Table 2 we give the $\mathrm{SiO}$ column density, $N(\mathrm{SiO})$, determined for each sample after the irradiation step. This information is used to evaluate the relative "hydroxylation" of the sample by dividing the variation of the $\mathrm{OH}$ column density by that of $\mathrm{SiO}$. If we consider the $\mathrm{OH}$ column density of the non irradiated sample (Almax4) as an indicator of atmospheric contamination to be subtracted from the column densities of the irradiated samples, we find relative hydroxylation of $\sim 0.3 \%, 1.5 \%$ and $2.8 \%$ for respectively Almax 1 $\left(3 \times 10^{16} \mathrm{H}^{+} / \mathrm{cm}^{2}\right)$, Almax $2\left(10^{17} \mathrm{H}^{+} / \mathrm{cm}^{2}\right)$ and Almax3 $(3 \times$ $\left.10^{17} \mathrm{H}^{+} / \mathrm{cm}^{2}\right)$. Once again, one can see that the relative hydroxylation in a sample increases with an increasing irradiation fluence but the evolution is not linear. This corroborates the idea that a maximal value of hydration by proton implantation into a "grain" exists and that it has a maximum of about $\sim 3 \%$.

\section{2. $\mathrm{SiH}$ band IR results}

The exact position of the SiH stretching band may vary from $2278 \mathrm{~cm}^{-1}$ in oxidized silicates to $2110 \mathrm{~cm}^{-1}$ in reduced solids

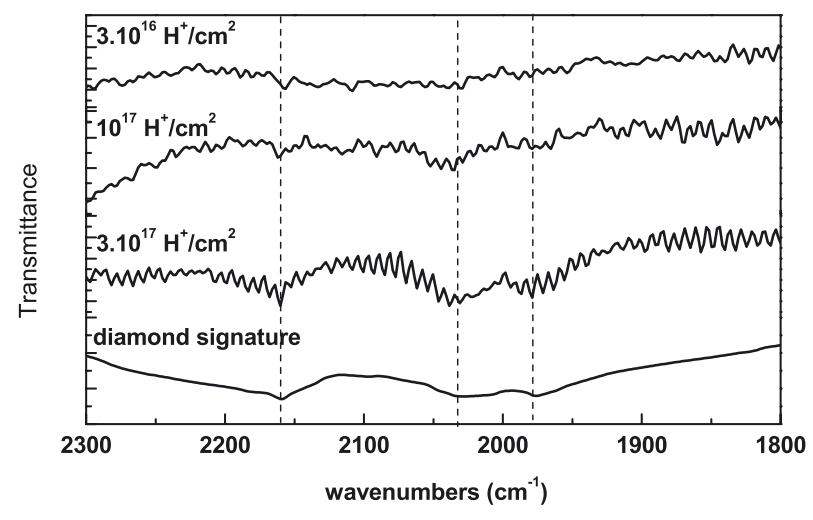

Fig. 3. The IR SiH band region for the three irradiated samples, compared to the diamond signatures in the same range. For each plot, the corresponding fluence is given on the left. For a rapid visual comparison we merged different plots and did not scale the transmittance axis.

(Moore et al. 1991; Nuth et al. 1992). In our experiments, the main difficulty in identifying the $\mathrm{SiH}$ band is due to the important diamond IR signature extending from 2700 to $1500 \mathrm{~cm}^{-1}$, corresponding to $\sim 3.7$ to $6.7 \mu \mathrm{m}$ (not reported here, see Fig. 1 in Djouadi et al. 2005). Despite the division of each sample spectrum by its corresponding diamond spectrum a slight residual signature of the diamond remains and makes it difficult to interpret the features. This is illustrated in Fig. 3 which gives the results of the reduced spectra in the range where the $\mathrm{SiH}$ signature is expected. For comparison, we merged the obtained spectra of the studied samples in the same plot. They are ranked by increasing fluence and we added the diamond signature in the same range. One can see that all the features observed in the spectra of the irradiated samples correspond to the diamond signatures as indicated by the dotted vertical lines in the plot. From this we determine that no visible $\mathrm{SiH}$ bond signature is seen in our irradiated samples, a not too surprising result considering the oxidizing environment offered by the silicate sample, which certainly favors $\mathrm{OH}$ formation over $\mathrm{SiH}$ formation, which probably also applies to grains in the ISM.

\subsection{NanoSIMS results}

In Fig. 4 the $\mathrm{H}$ concentrations are reported as $\mathrm{H}^{+}$counts per sec as a function of the cycle number $(1$ cycle $=5 \mathrm{~s})$. Although the data have not been calibrated absolutely, the $\mathrm{H}$ concentration is given by the count rate recorded by the electron multipliers. The correspondence between depth and cycle number is more tenuous because, as mentioned earlier, the removal of the surface is not a spatially homogeneous process. Figure 4 shows that the full thin film (initially $\approx 100 \mathrm{~nm}$ thick) is sputtered in $\approx 40 \mathrm{cy}$ cles. Thus, Fig. 4 gives the hydrogen concentrations in the samples as a function of depth, where we compare a non irradiated sample (Almax4) with the two samples irradiated with different fluences (Almax2 and Almax3). For the two samples Almax3 and Almax 4 we measured the $\mathrm{H}$ profile at two different spatial positions: on Almax3 to check the homogeneity of the $\mathrm{H}$ depth implantation and on Almax4 to check the homogeneity of the hydrogen (water) concentration in a non-irradiated sample.

In contrast to the "plateau" defined by Almax4 (the non irradiated sample), Almax 2 and Almax 3 (dashed and dotted lines respectively) exhibit different depth profiles. Almax 2 was subjected to a lower fluence of protons than Almax3. Therefore the difference between the patterns of Almax 2 and Almax 3 may be due to diffusion effects. Indeed the pattern for Almax 2 indicates 


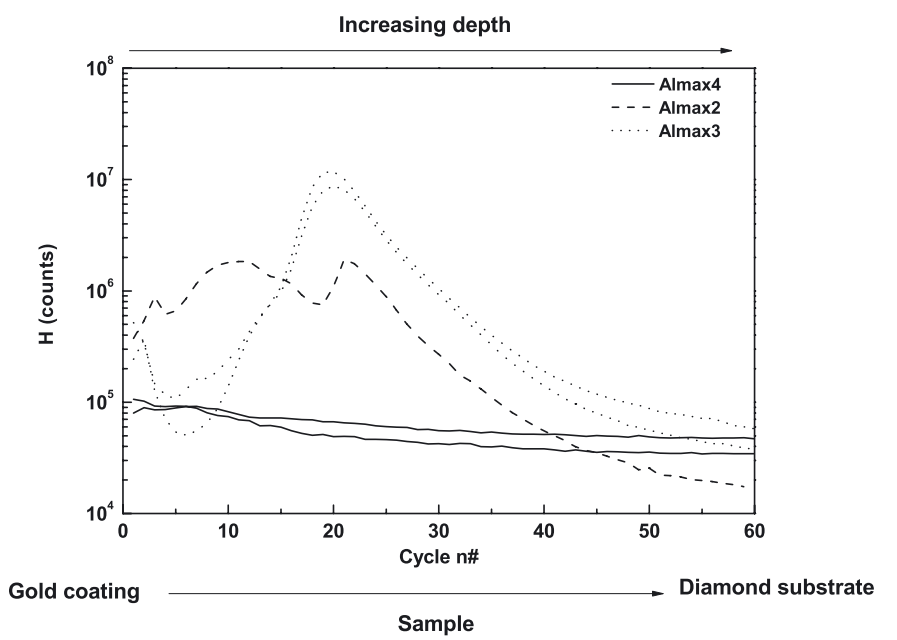

Fig. 4. $\mathrm{H}$ distribution within the samples plotted as $\mathrm{H}$ counts versus cycle number. For Almax3 and Almax4 the data for two different positions are shown. The inserted legend gives the reference for the different curves.
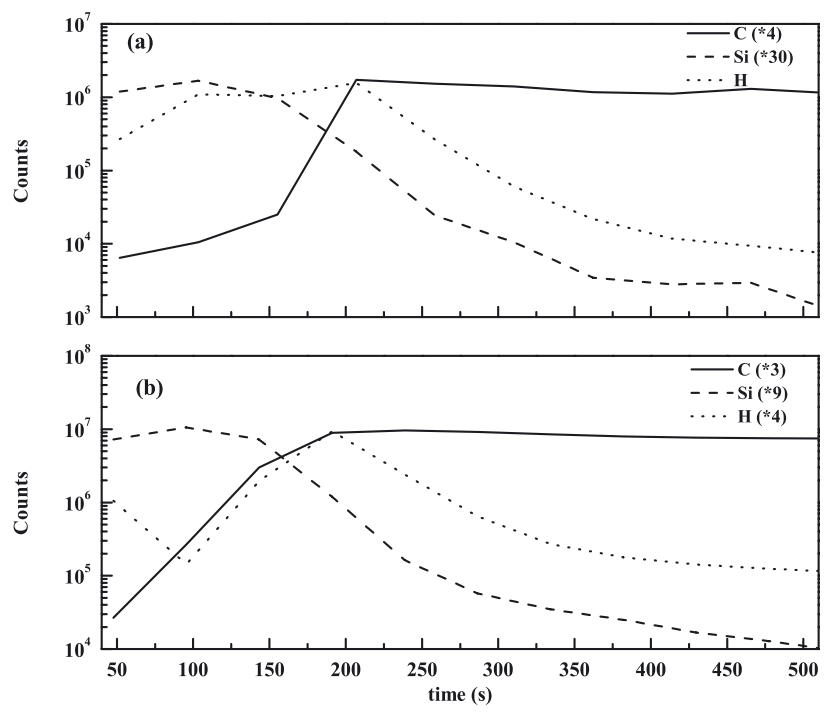

Fig. 5. The collected ${ }^{13} \mathrm{C},{ }^{29} \mathrm{Si}$ and $\mathrm{H}$ versus time for the Almax 2 a) and Almax 3 b) samples. To allow a comparison between the 3 profiles, the count rates for the analyzed elements have been rescaled, the multiplying factors are given in the top right in each panel.

a rather homogenous distribution of $\mathrm{H}$ in its layer while, in Almax3, H may have diffused towards the interface of the diamond substrate/silicate film. Such a diffusion may have been mediated by the structural damage caused by the higher fluence (a factor of three more) in Almax 3 compared with Almax2. In order to better support this interpretation, ${ }^{13} \mathrm{C},{ }^{29} \mathrm{Si}$ and $\mathrm{H}$ depth profiles for Almax 2 and Almax 3 were measured (see Fig. 5 panels (a) and (b) respectively). Note that we can also explain the difference between the two profiles by a redistribution in depth of the implanted $\mathrm{H}$ atoms in Almax3 due to the annealing treatment invoked in the experimental methods section.

The data are reported as a function of time (i.e., "depth"). The $\mathrm{H}$ count rate decreases sharply after the $\mathrm{C}$ reaches a plateau, which is the signature of the diamond substrate. Therefore, as postulated from Fig. 4, the peak in the hydrogen profiles corresponds to the substrate/silicate interface. In addition, the $\mathrm{Si}$ counts decrease before the $\mathrm{C}$ plateau, indicating a marked spatial heterogeneity at the bottom of the ion track. Indeed, on the ion

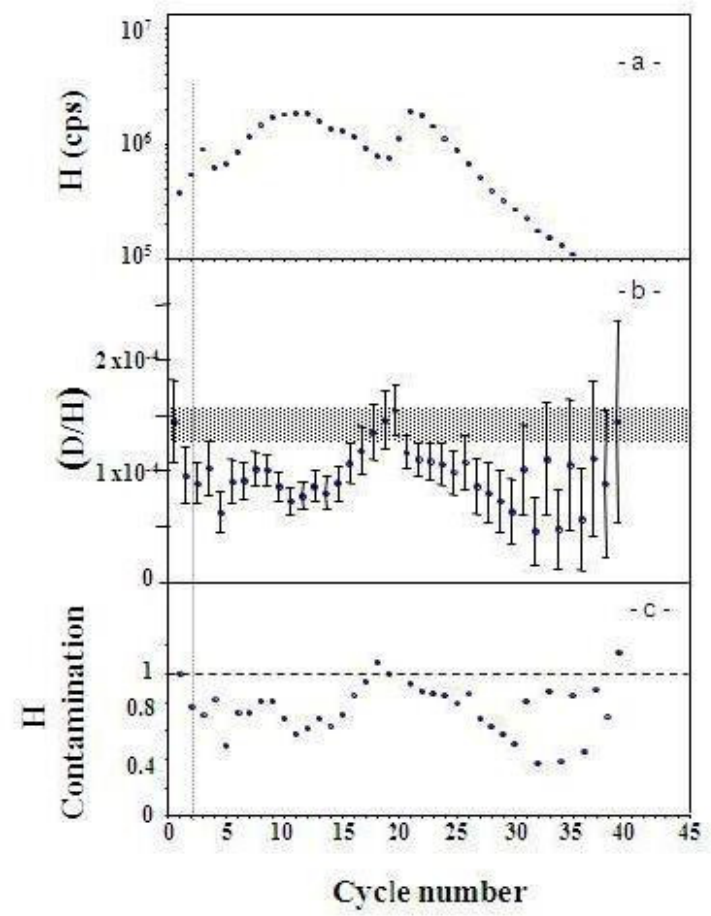

Fig. 6. Almax $2 \mathrm{H}$ concentration expressed in counts per second a), D/H ratio b) and the fraction of atmospheric moisture c) versus the sample depth expressed as the cycle number $(100 \mathrm{~nm} \approx 40$ cycles $)$. The $\mathrm{H}$ above the shaded area (in b) is ascribed to surface contamination (see the text).

images (not shown here) recorded during $\mathrm{C}$-Si analyses, small carbon surfaces gradually emerged in the silicon background during sputtering.

The $\boldsymbol{R}$ values defined in Sect. 2.4.2 are calculated from the results shown in Fig. 4 and are reported below in Table 3. In the $\boldsymbol{R}$ parameter, the absolute $\mathrm{H}$ concentration for each sample indicates the integrated count rate between cycle \#10 and \#50. With this procedure $\boldsymbol{R}$ gives the fraction of $\mathrm{H}$ atoms trapped in the sample during the irradiation.

In Figs. 6 and 7 we report the $\mathrm{H}$ concentration (-a-) as previously reported in Fig. 4, the $\mathrm{D} / \mathrm{H}$ ratio (-b-) and the fraction of atmospheric moisture (-c-), as a function of the cycle number for Almax 2 and Almax3. The contamination by atmospheric moisture (-c-) is calculated using the mass balance described in Sect. 2.4.2. In this calculation, it is assumed that hydrogen having a $\mathrm{D} / \mathrm{H}$ ratio lower than $125 \times 10^{-6}$ (the shaded area in -b-) contains a measurable fraction of implanted $\mathrm{H}$. In other words: $\mathrm{D} / \mathrm{H}$ values $>125 \times 10^{-6}, \mathrm{H}$ are of atmospheric origin, hence $\boldsymbol{x}=1$ in panel -c-. The integrated values of $\boldsymbol{x}$ are reported in Table 3 and give the fraction of atmospheric moisture-derived $\mathrm{H}$ introduced into the samples during irradiation. Note that, although this fraction is rather high $(\approx 60 \%)$, the concentration of implanted $\mathrm{H}$ in the irradiated samples is clearly detectable in the $\mathrm{D} / \mathrm{H}$ profile, which exhibits a minimum well below any reasonable values for natural or industrial contamination.

From the $x$ parameter we deduce the "NanoSIMS measured column density" (NanoSIMS C.D. in Table 3), which gives the number of $\mathrm{H}$ atoms actually implanted into the sample by irradiation $\left(\right.$ per $\mathrm{cm}^{2}$ ). We summarize these results in Table 3 .

Four important observations arise from Table 3: (1) the parameters $\boldsymbol{x}$ and $\boldsymbol{R}$ are quite different, $\boldsymbol{R}$ can be viewed as the $\mathrm{H}$ background carried by the silicate matrix while $\boldsymbol{x}$ indicates 


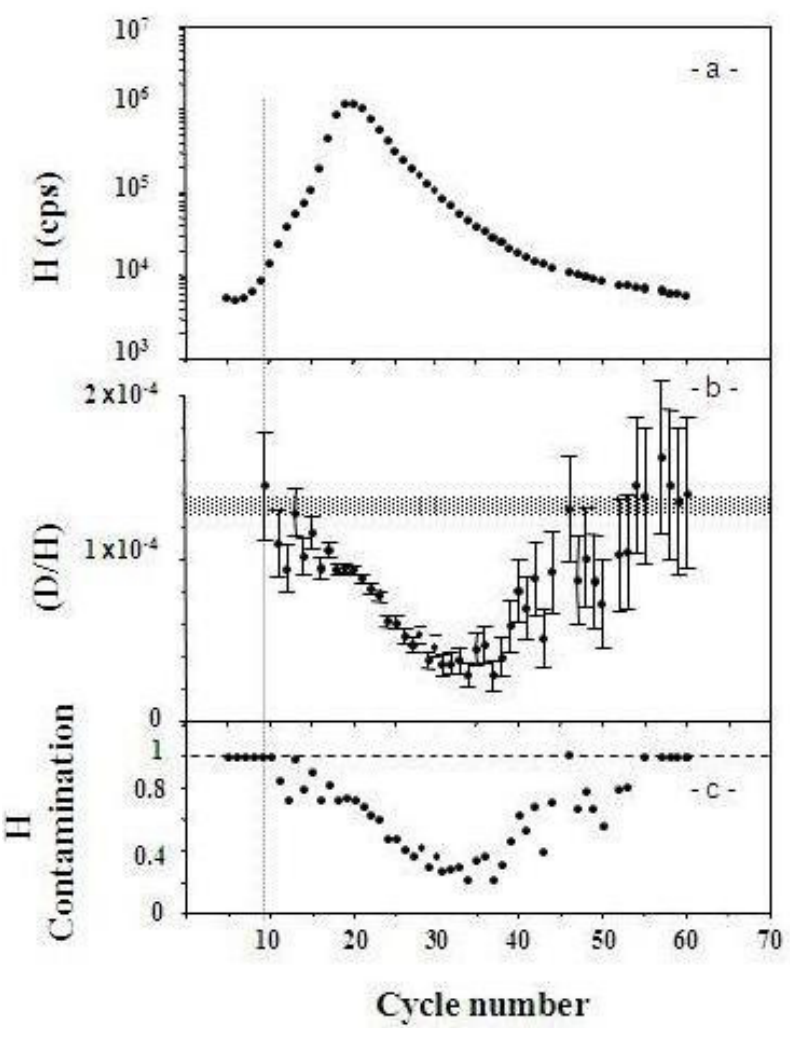

Fig. 7. Almax $3 \mathrm{H}$ concentration expressed in counts per second (-a-), $\mathrm{D} / \mathrm{H}$ ratio (-b-) and the fraction of atmospheric moisture (-c-) versus the sample depth expressed as the cycle number ( $100 \mathrm{~nm} \approx 40$ cycles). The $\mathrm{H}$ above the shaded area (in -b-) is ascribed to surface contamination (see the text).

Table 3. The NanoSIMS results for Almax2 and Almax3.

\begin{tabular}{lcccc}
\hline \hline $\begin{array}{l}\text { Sample } \\
\text { ref. }\end{array}$ & $\begin{array}{c}\mathrm{D} / \mathrm{H} \\
\times 10^{-5}\end{array}$ & $\begin{array}{c}\boldsymbol{x} \\
\%\end{array}$ & $\begin{array}{c}\boldsymbol{R} \\
\%\end{array}$ & $\begin{array}{c}\text { NanoSIMS C.D. } \\
\left(\mathrm{H} \text { atoms } / \mathrm{cm}^{2}\right)\end{array}$ \\
\hline Almax2 & $9.8 \pm 0.3$ & $71.5 \pm 3.5$ & $7.0 \pm 0.7$ & $1.72 \times 10^{15}$ \\
Almax3 & $8.3 \pm 0.2$ & $60.5 \pm 3.5$ & $2.9 \pm 0.6$ & $3.67 \times 10^{15}$ \\
\hline
\end{tabular}

Notes. The calculated contamination is given by $\boldsymbol{x}$, the measured contamination represented by $\boldsymbol{R}$ and the NanoSIMS-measured column density (NanoSIMS C.D. - see text for details).

an additional contamination taking place during or after the $\mathrm{H}$ implantation via an addition of $\mathrm{OH}$ of atmospheric origin or, and more likely, induced by irradiation damage in the matrix. From Table 3, it is worth noting that most of the atmospheric contamination is caused by the implantation procedure; (2) the deduced NanoSIMS column densities (using a neutral-ion conversion factor varying from 50/1 to 300/1) are within a factor of $\sim 1$ and 2 for Almax 2 and Almax3, respectively, equal to those calculated from IR data; (3) the NanoSIMS measured column density of Almax 3 is $\sim 2.1$ fold that of Almax 2 , in reasonably good agreement with the increase by a factor of 3 in the proton fluence for Almax3, compared with Almax2; (4) the fraction of implanted protons that induce an $\mathrm{OH}$ bond formation is rather low $(<2.0 \%$ cf. Table 4$)$ but is significant in terms of the total amount of hydroxyl groups formed in the amorphous silicates $(1-\boldsymbol{x} \approx 40 \%)$. The other implanted protons were assumed to be lost by diffusion and escaped from the sample, as $\mathrm{H}_{2}$ molecules. This effect was already observed in 2004 by Yoshida et al. (via FTIR spectroscopy) who investigated the hydrogen
Table 4. Comparison between NanoSIMS and IR results, where $F$ is the proton fluence $\left(\mathrm{H}^{+} / \mathrm{cm}^{2}\right)$ and the $\mathrm{OH}$ bond formation efficiency, $f_{\mathrm{OH}}$, is given by $N(\mathrm{OH}) / \mathrm{F}$.

\begin{tabular}{|c|c|c|c|c|c|}
\hline \multirow[b]{2}{*}{ sample } & \multirow[b]{2}{*}{$F$} & \multicolumn{2}{|c|}{ NanoSIMS results } & \multicolumn{2}{|c|}{ IR results } \\
\hline & & $\begin{array}{l}N(\mathrm{H}) \\
\left(\mathrm{cm}^{-2}\right)\end{array}$ & $f_{\mathrm{OH}}$ & $\begin{array}{l}N(\mathrm{OH}) \\
\left(\mathrm{cm}^{-2}\right)\end{array}$ & $f_{\mathrm{OH}}$ \\
\hline Almax 1 & $3 \times 10^{16}$ & - & - & $0.27 \times 10^{15}$ & $0.90 \%$ \\
\hline Almax2 & $\sim 10^{17}$ & $1.72 \times 10^{15}$ & $1.72 \%$ & $1.64 \times 10^{15}$ & $1.64 \%$ \\
\hline Almax3 & $3 \times 10^{17}$ & $3.67 \times 10^{15}$ & $1.22 \%$ & $2.65 \times 10^{15}$ & $0.88 \%$ \\
\hline
\end{tabular}

retention in proton irradiated silica glasses with different $\mathrm{OH}$ contents (Yoshida et al. 2004).

\subsection{IR versus NanoSIMS results}

In Table 4 we compare and summarize the IR and NanoSIMS results. The two analytical techniques indicate that less than $2 \%$ of the incident ions lead to the formation of $\mathrm{OH}$ bonds, yielding a relative hydroxylation of the sample of around 3\% (see Sect. 3.1).

As seen in Table 4, the number of the incident ions that form $\mathrm{OH}$ bonds in the target, deduced from the NanoSIMS data, are slightly greater than those deduced from the IR data. This could be explained by the fact that in NanoSIMS analyses all the $\mathrm{H}$ atoms present in the irradiated samples are detected, either in the form of trapped $\mathrm{H}_{2}$ or as $\mathrm{OH}$, while with IR spectroscopy we can only detect the $\mathrm{OH}$ bonds (via $\sim 3500 \mathrm{~cm}^{-1}$ region).

\section{Discussion}

The observation of the $\mathrm{SiH}$ band, expected in the 2278 to $2110 \mathrm{~cm}^{-1}$ region $(4.4-4.7 \mu \mathrm{m})$, was reported for the first time by Soifer et al. (1979) in W33A via the $4.6 \mu \mathrm{m}$ absorption band, and was also discovered in the line of sight to other embedded protostellar sources (Tegler et al. 1995, and references therein). However, the identification of the band with solid $\mathrm{CO}$ is now beyond any doubt.

Nuth \& Moore (1988) investigated the formation of $\mathrm{SiH}$ groups resulting from irradiation; they showed that proton irradiation of ice mixtures containing silicon hydride compounds show a $4.6 \mu \mathrm{m}$ stretching feature which is characteristic of the $\mathrm{SiH}$ group. They thus suggested that the implantation of hydrogen in supernova shocks could be a source of $\mathrm{SiH}$ formation in silicates. It could also be responsible for the $\mathrm{SiH}$ stretching band on planetary objects (Moore et al. 1991).

Previous irradiation experiments on silicates provided conflicting results. Buemi et al. (1994) failed to detect $\mathrm{SiH}$ groups in silicates (quartz, feldspar and palagonite) irradiated with $7.5 \times 10^{17} \mathrm{H}^{+}(1.5 \mathrm{keV}) / \mathrm{cm}^{2}$, but succeeded in silicon wafers where protons probably find interstitial sites to bond with $\mathrm{Si}$ atoms. Our results indicate that it is not possible to attach hydrogen atoms to silicon in an oxidizing material $(\mathrm{SiO})$ thus rejecting the previous ideas from Nuth \& Moore (1988).

Experimentally, regarding the formation of $\mathrm{OH}$ groups under $\mathrm{H}$ irradiation, Bradley (1994) found hydroxyl formation in olivine irradiated at $20 \mathrm{keV}$. The $\mathrm{OH}$ groups could form by ion exchange with cations in octahedral sites ( $\mathrm{Mg}$ or $\mathrm{Fe})$. If this reaction occurs during irradiation, this is of importance because it induces stiffness into the glass network. This aspect was already addressed by Jäger et al. (2003) who synthesized silicates by the sol-gel method. The residual $\mathrm{OH}$ groups in the silicate network strongly influence the ability for crystallization at lower 
temperatures. Jäger et al. (2003b) showed that the threshold for crystallization was lowered by $200-300 \mathrm{~K}$ with important consequences for the incorporation of silicate dust into protoplanetary disks.

The detection of $\mathrm{OH}$ in silicates in dense molecular cloud environments is practically impossible because the $\mathrm{OH}$ stretch band occurs at about $2.7 \mu \mathrm{m}$ and is masked by the strong water ice band at $2.7-3.5 \mu \mathrm{m}$.

In the diffuse ISM (DISM), a possible identification was made by Whittet et al. (1997) who detected a weak feature near $2.75 \mu \mathrm{m}$ in IR spectra of dust towards Cygnus OB2 No. 12. Figure 2 in Whittet et al. (1997), compares the spectra of this source obtained with the Kuiper Airbone Obervatory (KAO) by Knacke et al. (1985), to those obtained with ISO at two resolving powers $R(R=200$ and 400). The three spectra reported there exhibit a feature centered at $\sim 2.75 \mu \mathrm{m}$.

Whittet et al. (2001) re-reduced their earlier data and concluded that the silicate $\mathrm{OH}$ feature is not convincingly present in the re-reduced spectrum but they do not show the new spectrum and do not comment on the KAO spectrum shown in their previous article. We thus estimate that the absence of hydrated silicates in the observations could be due to the procedures of data reduction, always difficult for broad bands, which could mask the very weak features that may indeed be present.

To compare these observational data to our experimental results we use the optical depth $\left(\tau_{2.75}=0.035 \pm 0.010\right)$ and the full width at half maximum of the band $(0.15 \mu \mathrm{m})$ that Whittet et al. (1997) extracted from their data. Given the above-mentioned incertainties in the interpretation of these data we use them as upper limits on the hydration of interstellar silicates. We thus estimate an $\mathrm{OH}$ column density, $N(\mathrm{OH})$, using the band strength $A=2.8 \times 10^{-16} \mathrm{~cm} \mathrm{~mol}^{-1}$ for the $\mathrm{OH}$ bond, by:

$N(\mathrm{OH}) \sim(\tau \times F W H M) / A \sim 2.5 \times 10^{16} \mathrm{OH} / \mathrm{cm}^{2}$.

From the silicate column density towards the same source, given by Bowey et al. $(1998), N($ silicate $)=1.5 \times 10^{18}$ molecules $/ \mathrm{cm}^{2}$, we determine an upper limit to the "relative hydration" $(N(\mathrm{OH}) / N($ silicate $))$ of the dust in this source of $\sim 1.7 \%$.

Note that this result is in very good agreement with the values determined from our experimental study: a relative hydroxylation around $3 \%$.

In interstellar supernova-generated shocks in the ISM the implanted proton mass should be of the order of the grain mass (e.g., Jones et al. 1994) and, as such, would be expected to leave an observable trace on the silicates. The lack of an observed $\mathrm{SiH}$ band and the observed limits on $\mathrm{OH}$ in silicates in the ISM $(<2 \%)$ are therefore consistent with our results, which show that $\mathrm{H}$ is inefficiently incorporated into silicates during irradiation.

According to our findings, the $\mathrm{OH}$ band is present at a level of only $\sim 3 \%$ of that of the $\mathrm{SiO}$ band. Although a priori small, this concentration corresponds to several types of whole rock carbonaceous chondrites (hereafter referred to as CC and includes most IDPs) that are often invoked to be the carriers of water on Earth since they are the only water-rich extraterrestrial objects currently observed in the Solar System having, on average, a D/H ratio similar to the Earth's (Robert et al. 2000). As far as the origin of water on Earth is concerned, two questions need to be answered: (1) how was this water introduced to the primitive Earth? (2) How were the silicates (clays) hydrated in the early Solar System?

"Wet" and "dry" accretion models have been proposed in the literature (see Drake 2005, for a discussion). In "wet" accretion models, hydroxylated silicates move inward along solar disk to the Earth forming regions (Ciesla \& Lauretta 2005), accrete in the form of water-rich parent bodies and are then embedded into the proto-Earth. In the "dry" models, also called "exogeneous" scenario, hydroxylated silicates are added to the dry silicates of the terrestrial primitive mantle via one (or several) collisions with CC like planetoids (1000 km in diameter), occuring at the end of the Earth's accretion. Although there is no clear consensus in the literature, "dry" models are in clear agreement with the geochemical data (Javoy 1995; Robert 2001) or with dynamical simulations (Morbidelli et al. 2000; Lunine et al. 2007). As far as the mineralogical carrier of water in planetary bodies is concerned, the present results re-open the interesting possibility, related to question (2), that the silicates were hydroxylated prior to their incorporation into CC parent bodies.

In cosmochemistry, the general consensus for the origin of clay minerals in CCs can be summarized by a two-step process: (1) ice grains were embedded in meteorite parent bodies and (2) a rise in temperature of the parent body, caused by the release of accretional energy and/or from radioactivity, produced a circulation of liquid water that leached the pre-existing dry silicates, yielding the fomation of clays. Although this scenario is difficult to reconcile with the isotopic heterogeneity observed in the $\mathrm{D} / \mathrm{H}$ ratio of some chondrites (Deloule \& Robert 1995), it remains the most popular one since the alteration kinetics exclude hydroxylation of crystallized silicates in the gas phase.

The present results allow us to overcome the kinetic limitation since they demonstrate that the hydroxylation can take place in the interstellar medium due to the low-energy proton irradiation of amorphous silicates in shock waves. If this is so, most clays would have been present in the protosolar disk in the form of sub-micrometric amorphous particles whose movements were coupled with the gas.

This scenario can be tested in the laboratory based on the determination of the isotopic fractionation taking place during the implantation of $\mathrm{H}$ and D. Indeed, meteorite clay minerals, as well as Earth's oceans, have a distinct hydrogen isotopic signature $\left(\mathrm{D} / \mathrm{H}=(1.5 \pm 0.5) \times 10^{-4}\right)$ compared (i) with the solar wind $(\mathrm{D} / \mathrm{H}=0)$ (ii) with the molecular hydrogen of the protosolar disk $\left(\mathrm{D} / \mathrm{H}=(2.5 \pm 0.5) \times 10^{-5}\right)$ (iii) with the present day interstellar $\mathrm{H}\left(\mathrm{D} / \mathrm{H}=(1.5 \pm 0.5) \times 10^{-5}\right)$ or (iv) with deuterated interstellar water $\left(\mathrm{D} / \mathrm{H} \sim 3 \times 10^{-2}\right.$, Butner et al. 2007).

Therefore, in order to test the scenario of the hydroxylation of amorphous silicates through $\mathrm{H}$ implantation, the implantation of $\mathrm{D}$ should yield an increase of the implanted $\mathrm{D} / \mathrm{H}$ ratio by a factor of $\sim 6$ up to 10 i.e. an increase from $\mathrm{D} / \mathrm{H}=(1.5 \pm 0.5) \times$ $10^{-5}$ to $\mathrm{D} / \mathrm{H}=(1.5 \pm 0.5) \times 10^{-4}$. Although such a huge isotopic fractionation would be surprising, we plan to investigate this in the laboratory.

\section{Conclusions}

Our coordinated study, including IR spectroscopy and NanoSIMS analysis, clearly establishes that $H$ irradiation can lead to the formation and storage of hydroxyl groups in amorphous silicates (olivine-type).

Saturation levels for the $\mathrm{OH}$ groups of about 3\%, compared to $\mathrm{SiO}$ in the silicates combined with the "inefficient" formation of $\mathrm{OH}$ bonds during proton irradiation (less than $2 \%$ of the incident ions are found to form $\mathrm{OH}$ ), are entirely consistent with the lack of an observed $\mathrm{OH}$ stretching feature in the diffuse interstellar medium. Further, our work indicates that the implantation of $\mathrm{H} / \mathrm{H}^{+}$into silicates in interstellar shocks will not leave a readilyobservable trace of these irradiations. 
Given that hydrogen is ubiquitous in the ISM and that silicates strongly interact with the gas in shock waves, a low-energy proton irradiation of silicates is a way to form stable hydroxyl radicals. If such is the case, then amorphous silicates could be $\mathrm{OH}$ reservoirs, which could be produced in the gas phase of the protosolar disk or even inherited from the interstellar medium. However, a deuterium enrichment relative to solar or to the interstellar hydrogen $\mathrm{D} / \mathrm{H}$ ratio, is a characteristic feature of chondritic hydroxyls as well as terrestrial oceans. Therefore, the validity of the presently proposed model relies on an isotopic fractionation that could possibly have taken place during hydrogen implantation. Such a fractionation will be tested by subsequent laboratory studies.

Acknowledgements. The authors would like to thank the referee Joseph Nuth and the Editors for their comments and suggestions. We would also like to thank Dominique Le Du for having performed the irradiation experiments at the Centre de Spectrométrie Nucléaire et de Spectrométrie de Masse of the University of Orsay and the student Severine Pouchot-Camoz-Gandome who contributed to the experimental work. This work has been partly funded by the French National Program of Planetology (PNP-CNRS), the author Z. Djouadi is grateful for this support.

\section{References}

Boss, A. P. 1998, Ann. Rev. Earth Planet. Sci., 26, 26

Bowey, J. E., Adamson, A. J., \& Whittet, D. C. B. 1998, MNRAS, 298, 131

Bradley, J. P. 1994, Science, 265, 925

Bradley, J. P., Keller, L. P., Snow, T. P., et al. 1999, Science, 285, 1716

Bradley, J. P., Dai, Z. R., Erni, R., et al. 2005, Science, 307, 244

Bregman, J. D., Witteborn, F. C., Allamandola, L. J., et al. 1987, A\&A, 187, 616

Brownlee, D., Tsou, P., Aléon, J., et al. 2006, Science, 314, 1711

Brucato, J. R., Strazzulla, G., Baratta, G., \& Colangeli, L. 2004, A\&A, 413, 395

Buemi, A., Cimino, G., Leto, G., \& Strazzulla, G. 1994, Icarus, 108, 169

Butner, H. M., Charnley, S. B., Ceccarelli, C., et al. 2007, ApJ, 659, L137

Campins H., \& Ryan E. V. 1989, ApJ, 341, 1059

Carrez, P., Demyk, K., Cordier, P., et al. 2002, Meteor. Planet. Sci., 37, 1599
Chauvin, N., Dayras, F., Le Du, D., \& Meunier, R. 2004, Nucl. Inst. Meth., A521, 149

Ciesla, F., \& Lauretta, D. 2005, Earth Planet. Sci. Lett., 231, I 1

Crovisier, J., Leech, K., Bockelée-Morvan, D., et al. 1997, Science, 275, 1904

Deloule, E., \& Robert, F. 1995, Geochim. Cosmochim. Acta, 59, 4695

Demyk, K., Carrez, Ph., Leroux, H., et al. 2001, A\&A, 368, L38

Demyk, K., d'Hendecourt, L., Leroux, H., et al. 2004, A\&A, 420, 233

d'Hendecourt, L. B., \& Allamandola L. J. 1986, A\&AS, 64, 453

Djouadi, Z., d'Hendecourt, L., Leroux, H., et al. 2005, A\&A, 440, 179

Drake, M. J. 2005, M\&PS, 40, 1

Jag̈er, C., Fabian, D., Schrempel, F., et al. 2003a, A\&A, 401, 57

Jag̈er, C., Dorschner, J., Mutschke, H., et al. 2003b, A\&A, 408, 193

Javoy, M. 1995, Geophys. Res. Lett., 16, 2219

Jones, A. P., Tielens, A. G. G. M., Hollenbach D. J., et al. 1994, ApJ, 433, 797

Jones, A. P., Tielens, A. G. G. M., \& Hollenbach, D. J. 1996, ApJ, 469, 740

Kemper, F., Vriend, W. J., \& Tielens, A. G. G. M. 2004, ApJ, 609, 826

Knacke, R. F., Puetter, R. C., Erickson, E., et al. 1985, AJ, 90, 1828

Li, A., \& Draine, B. T. 2001, ApJ, 550, L213

Malfait, K., Waelkens, C., Bouwman, J., et al. 1999, A\&A, 345, 181

Mathis, J. S. 1998, ApJ, 497, 824

Matrajt, G., Muñoz Caro, G. M., Dartois, E., et al. 2005, A\&A, 433, 979

Messenger, S., Keller, L. P., Stadermann, F. J., et al. 2003, Science, 300, 105

Molster, F. J., Waters, L. B. F. M., \& Tielens, A. G. G. M. 2002, A\&A, 382, 222

Moore, M. H., Tanabé, T., \& Nuth, J. A. 1991, ApJ, 373, L31

Morbidelli, A., Chambers, J., Lunine, J. I., et al. 2000, M\&PS, 35, 1309

Nuth, J. A., \& Moore, M. H. 1988, ApJ, 329, L113

Nuth, J. A., Moore, M. H., \& Tanabé, T. 1992, Icarus, 98, 207

Robert, F. 2001, Science, 293, 1056

Robert, F., Gautier, D., \& Dubrulle, B. 2000, Space Sci. Rev., 92, 201

Soifer, B. T., Puetter, R. C., Russell, R. W., et al. 1979, ApJ, 232, L53

Sylvester, R. J., Kemper, F., Barlow, M. J., et al. 1999, A\&A, 352, 587

Tegler, S. C., Weinstraub, D. A., Rettig, T. W., et al. 1995, ApJ, 439, 279

Timmermann, R., \& Larson, H. P. 1993, ApJ, 415, 820

Thomas, K. L., Blanford, G. E., Keller, L. P., et al. 1993, Geochim. Cosmochim. Acta, 57, 1551

Waelkens, C., Waters, L. B. F. M., de Graauw, M. S., et al. 1996, A\&A, 315, L245

Waters, L. B. F. M., Molster, F. J., de Jong, T., et al. 1996, A\&A, 315, L361

Whittet, D. C. B., Boogert, A. C. A., Gerakines, P. A., et al. 1997, ApJ, 490, 729

Whittet, D. C. B., Pendleton, Y. J., Gibb, E. L., et al. 2001, ApJ, 550, 793

Yoshida, T., Tanabe,T., Hirano, M., et al. 2004, Nucl. Inst. Methods Phys. Res., B218, 202 